\title{
Some Properties of prominence eruption associated CMEs during 1996-2009
}

\author{
Nishant Mittal ${ }^{1,2}$ \\ ${ }^{1}$ Astrophysics Research Group, Meerut College, Meerut-250001, India \\ email: nishantphysics@yahoo.com \\ ${ }^{2}$ Dept. of Physics, Krishna Institute of Management and Technology, Moradabad-244001, \\ U. P., India
}

\begin{abstract}
Solar prominences can be viewed as pre-eruptive states of coronal mass ejections (CMEs). Eruptive prominences are the phenomena most related to CMEs observed in the lower layers of the solar atmosphere. We have made a comprehensive statistical study on the CMEs associated with prominence eruptions. We have examined the distribution of CMEs speed and acceleration for prominence eruptions associated CMEs. We also examine the speed-acceleration correlation for these events and there is no correlation between speed and acceleration. The mean angular width is almost similar to normal CMEs. The number variation during solar cycle of prominence activities is similar to the sunspot cycle.
\end{abstract}

Keywords. Sun; coronal mass ejections; prominence eruptions;

\section{Introduction}

CMEs are often seen as spectacular eruptions of matter from the Sun, which propagate outward through the heliosphere and often interact with the Earth's magnetosphere (Hundhausen 1997; Mittal \& Narain 2010 \& references there in). It is well known that these interactions can have substantial consequences on the geomagnetic environment of the Earth, sometimes resulting in damage to satellites (Mittal et al. 2010. The "classical" structure of a CME consists of three parts and one of these parts, a bright core, is the remnant of an eruptive prominence (Crifo et al. 1983; Illing \& Hundhausen 1985; Hundhausen 1999 and Gopalswamy et al. 2006, Mittal \& Narain 2010).

\section{Data and results}

Over the past 14-years the SOHO/LASCO instrument has been detecting CMEs. The LASCO instrument (Brueckner et al. 1995) consists of three coronagraphs C1, C2 and C3 that span the fields of view $1.1-3 \mathrm{R}_{\odot}, 2-6 \mathrm{R}_{\odot} \mathrm{S}$ and $4-30 \mathrm{R}_{\odot}$, respectively. The height-time data of CMEs used in this study are taken from the online SOHO/LASCO CME catalogue (http://cdaw.gsfc.nasa.gov/CME_list). The Prominence Eruption data has been taken from Nobeyama Radioheliograph; which detect 396 events during the period. The distribution of Prominence Eruption associated CMEs has been shown in Figure 1. It seems that the peak of the histogram occur at $350 \mathrm{~km} \mathrm{~s}^{-1}$; where the tail of large speeds is very short. The overall median (average) speed 447 (511) $\mathrm{km} \mathrm{s}^{-1}$; respectively. Figure 2 is the histogram of apparent angular width for the period 1996-2009. The average width from the 14-year data for Prominence Eruption associated CMEs (390) is 670 and the median width is 550 . Figure 3 is the histogram of CME acceleration for the period 1996-2009. It is clear from this figure that a majority (55\%) of Prominence Eruption associated CMEs are decelerated, about $12 \%$ of them move with little acceleration and the 


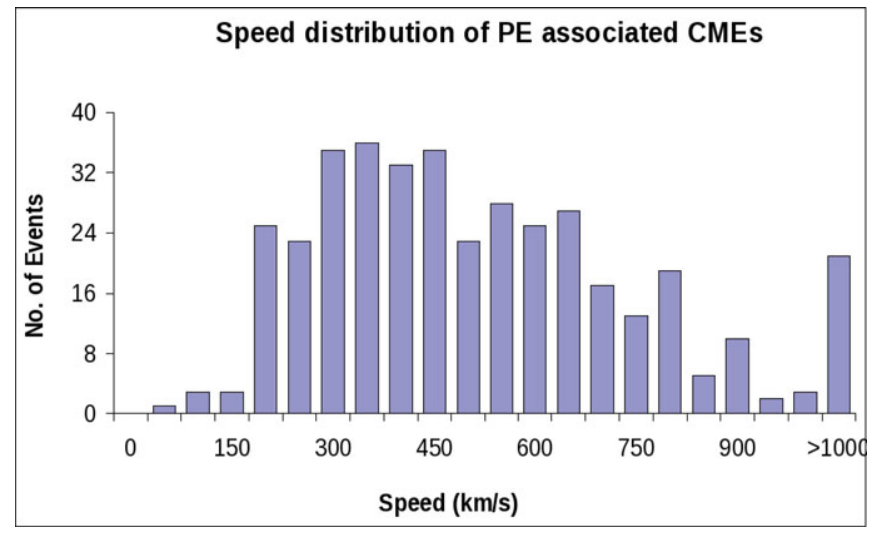

Figure 1. Histogram shows the speed distribution of Prominence Eruption associated CMEs during 1996-2009.

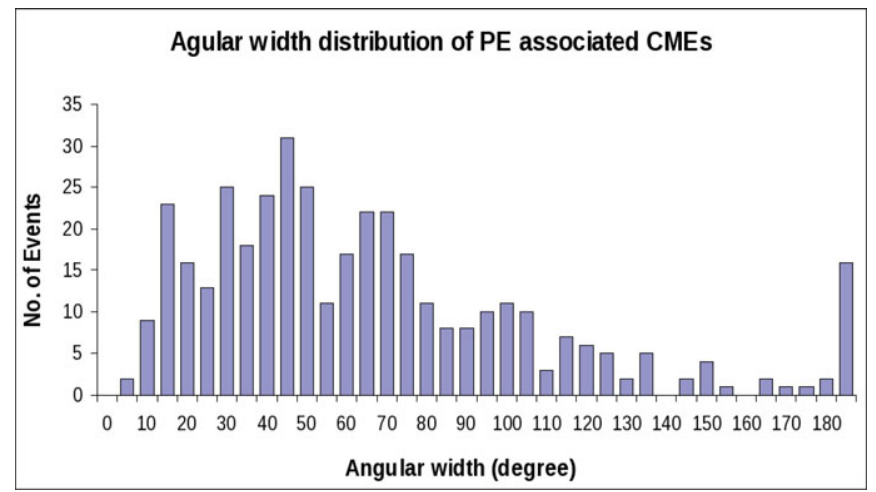

Figure 2. The width distribution of SOHO/LASCO Prominence Eruption associated CMEs from 1996 to 2009. The last bin shows all CMEs with width ¿1800, which amounts to 5-6\% of all Prominence Eruption associated CMEs.

remaining $33 \%$ have positive acceleration. Thus Prominence Eruption associated CMEs have clear bias towards deceleration. Figure 4 shows a scatter plot between the measured acceleration, a (in $\mathrm{m} \mathrm{s}^{-2}$ ) and speed, V (in $\mathrm{km} \mathrm{s}^{-1}$ ) of Prominence Eruption associated CMEs for which the acceleration estimate was possible.

Figure 5 exhibits total CMEs and Prominence Eruptions. It is clear from the figure that the solar maximum peak occurs at the year 2000 and 2002. Occurrence rate of Prominence Eruptions follows the solar cycle variation whereas CMEs occurrence rate does not follow the solar cycle variation.

\section{Conclusion}

In this paper, I have made a comprehensive statistical study on Prominence Eruption associated CMEs from 1996 to 2009. During the period 1996-2009 more than 14000 CMEs observed by SOHO/LASCO, whereas Nobeyama Radioheliograph detect 396 Prominence Eruption events, out of which 390 Prominence Eruptions are associated with CMEs.

1. Figure 1 shows that the Prominence Eruption associated CMEs have median (average) speed $447(511) \mathrm{km} \mathrm{s}^{-1}$. The number of CMEs having speeds greater than $1000 \mathrm{~km}$ $\mathrm{s}^{-1}$ is quite small $(5-6 \%)$. 


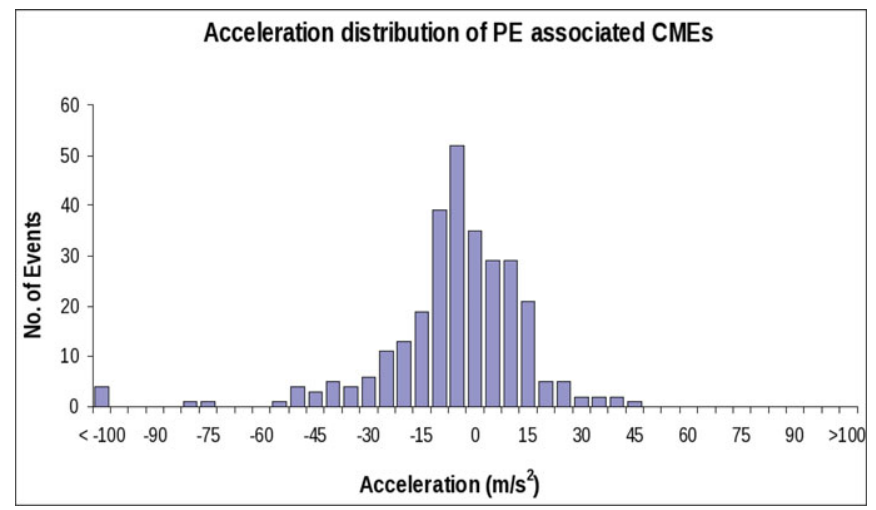

Figure 3. Histogram of acceleration of Prominence Eruption associated CMEs, showing clear bias towards deceleration.

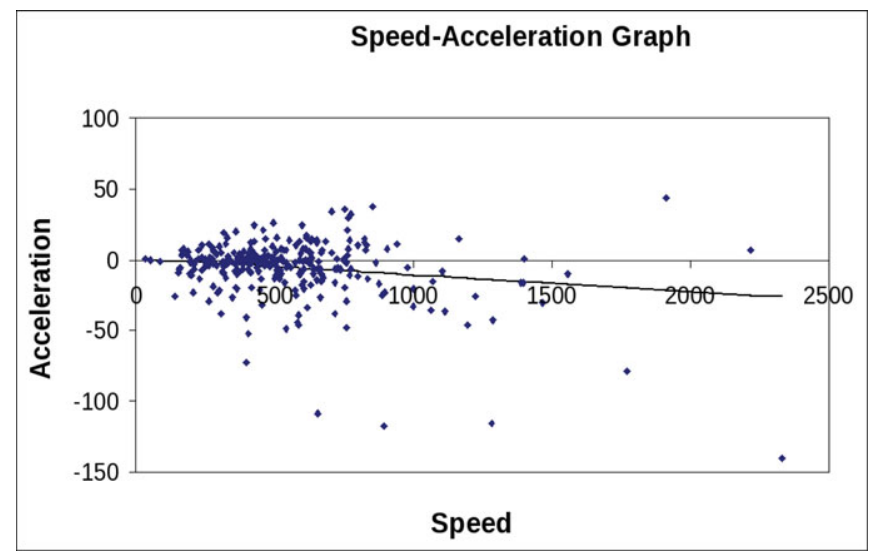

Figure 4. Acceleration as a function of speed of Prominence Eruption associated CMEs from 1996 to 2009. The acceleration has a large scatter, but there is a clear trend that the fast CMEs decelerate, while slow CMEs accelerate.

2. The average width for Prominence Eruption associated CMEs (390) is 670 and the median width is 550 .

3. The acceleration distribution shows a conspicuous peak near $-5 \mathrm{~m} \mathrm{~s}^{-1}$, about $55 \%$ have negative acceleration, $33 \%$ have positive acceleration and the remaining $12 \%$ have very little acceleration. The Prominence Eruption associated CME distribution is biased towards deceleration.

4. Rate of Prominence Eruptions varies with solar cycle.

\section{Acknowledgement}

Author is highly thankful to IAU and LOC/SOC of IAU S274 for providing financial help to attend the meeting. Author is thankful to KIMT, Moradabad authorities for their help and encouragement. I am also thankful to Pankaj Kumar for his useful suggestions. The CME catalog is generated and maintained at the CDAW Data Center by NASA and The Catholic University of America in cooperation with the Naval Research Laboratory. 


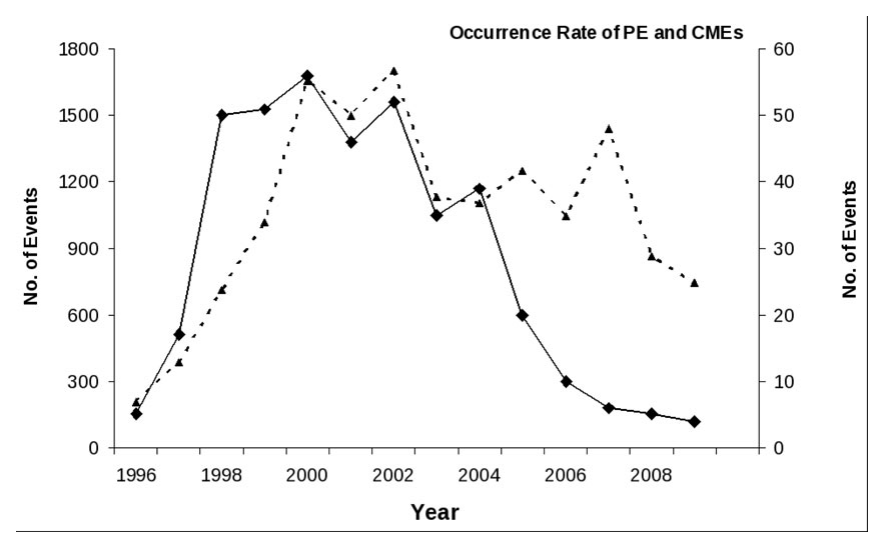

Figure 5. Comparison between annual CME occurrence rate and Prominence Eruptions from 1996-2009.

SOHO is a project of international cooperation between ESA and NASA. Author is also thankful to Nobeyama Radioheliograph for providing the prominence eruption data

\section{References}

Mittal, N. \& Narain, U. 2010, Journal of Atmospheric and Solar-Terrestrial Physics, 72, 643 Crifo, F., Picat, J. P., \& Cailloux, M. 1983, Sol. phys, 83, 143

Gopalswamy, N., Mikić, Z., Maia, D., Alexander, D., Cremades, H., Kaufmann, P., Tripathi, D., \& Wang, Y.-M. 2006, SSR, 123, 303

Hundhausen, A. 1999, The many faces of the sun: a summary of the results from NASA's Solar Maximum Mission., 143

Hundhausen, A. J., Burkepile, J. T., \& St. Cyr, O. C. 1994, JGR, 99, 6543

Illing, R. M. E. \& Hundhausen, A. J. 1985, JGR, 90, 275 